\title{
Évaluation de la dose à la peau en radiologie interventionnelle par l'utilisation de films radiochromiques
}

\author{
M. LABATTU ${ }^{1}$, J. GUERSEN ${ }^{1}$, V. CHASSIN ${ }^{2}$, L. CASSAGNES ${ }^{1}$, F. MAGNIER ${ }^{2}$, \\ D. DONNARIEIX ${ }^{2}$, L. BOYER ${ }^{1}$, P. CHABROT ${ }^{1}$
}

(Manuscrit reçu le 21 mai 2012, accepté le 28 août 2012)

RÉSUMÉ La directive 97/43/EURATOM du 30 juin 1997 pointe la radiologie interventionnelle comme une des pratiques spéciales (Article 9) susceptibles de délivrer des doses élevées, et pour lesquelles il est demandé de veiller à ce que les équipements, les procédures, les programmes d'assurance qualité et la formation des opérateurs soient particulièrement appropriées. En effet, l'utilisation d'un rayonnement $X$ de faible énergie lors de procédures allant de quelques secondes à plusieurs heures pour les examens complexes, renvoie inévitablement à des risques stochastiques et déterministes. L'évaluation de la dose maximum à la peau est donc une donnée appréciable dans l'objectif d'un suivi dermatologique du patient. Dans cette étude nous avons choisi d'utiliser comme détecteur les films radiochromiques XR-RV3Gafchromic $^{\circledR}$ afin d'obtenir une cartographie de la dose à la peau reçue par le patient lors d'une procédure. Les films radiochromiques sont intéressants car ils permettent à la fois de visualiser et de quantifier la région de dose maximale. Nous présentons ici les étapes préliminaires à une utilisation sur patient des films, à savoir l'étude des caractéristiques physiques, l'étalonnage en dose, la lecture par scanner plat et l'exploitation des films avec le logiciel de traitement d'images ImageJ. Les résultats obtenus montrent que l'utilisation des films radiochromiques est pertinente pour une évaluation dosimétrique sur patient. Plus particulièrement, la réponse des films diffère de moins de $3,6 \%$ entre l'étalonnage à $80 \mathrm{kV}$ et $120 \mathrm{kV}$ et l'incertitude globale reste inférieure à $20 \%$ jusqu'à une dose de $12 \mathrm{~Gy}$. L'évolution de la réponse du film dans le temps étant négligeable, la lecture peut être faite immédiatement et l'exploitation du film nécessite moins de 15 minutes pour obtenir la cartographie de la dose. L'application de cette technique de mesure in-vivo pour 58 procédures a permis de mettre en évidence une dose maximale à la peau supérieure à $3 \mathrm{~Gy}$ pour 12 patients. Ces patients ont alors été orientés vers un suivi dermatologique. Parmi ces patients, l'évaluation de la dose peau à partir des indicateurs dosimétriques fournis par l'installation aurait mené au suivi de seulement 4 patients.

ABSTRACT Assessment of skin dose in interventional radiology using radiochromic films.

The 97/43 /EURATOM Directive (June 30, 1997) indicates interventional radiology as a special practice (Article 9) able to deliver high doses, which is why having safety equipment and procedures, and appropriate quality assurance programs and training is essential. Indeed, using low-energy X-rays during procedures, ranging

CHU Clermont Ferrand, Pôle Imagerie Médicale, 58 rue Montalembert, 63003 Clermont-Ferrand Cedex 1, France.

Centre de Lutte contre le Cancer Jean Perrin, Service de Physique Médicale, 58 rue Montalembert, 63011 ClermontFerrand Cedex 1, France. 


\begin{abstract}
from a few seconds to several hours (complex cases), inevitably involves stochastic and deterministic risks. The evaluation of the maximum skin dose is useful data in the dermatological follow-up of the patient. In this study, we chose to use Gafchromic $^{\circledR}$ XR-RV3 radiochromic film as a detector to obtain a mapping of the skin dose received by the patient during a procedure. Radiochromic films are interesting because they allow one both to visualize and to quantify the region of the maximum dose. We present the preliminary stages of using the films on patients, that is to say, the study of physical characteristics, the dose calibration, the reading by flatbed scanner and the utilization of films with the image processing software ImageJ. The results show that the use of radiochromic films is relevant to a patient dosimetry assessment. Specifically, the films' response differs by less than $3.6 \%$ between calibration at 80 and $120 \mathrm{kV}$ and the overall uncertainty remains below $20 \%$ up to a dose of $12 \mathrm{~Gy}$. The evolving response of the films in time being negligible, the reading can be done immediately and utilizing the film requires less than 15 minutes to obtain the dose mapping. The application of this measurement technique in vivo for 58 procedures showed a maximum skin dose that exceeded $3 \mathrm{~Gy}$ for 12 patients. These patients were then sent to a dermatological follow-up. Among the 58 patients, assessment of the skin dose with the dosimetry indicators provided by the facilities would have led to the follow-up care of only 4 patients.
\end{abstract}

Keywords: Skin dose / interventional radiology / deterministic effect / radiochromic film

\title{
Introduction / contexte
}

La radiologie interventionnelle, dans sa définition la plus générale, associe simultanément une technique d'imagerie et un geste invasif à visée diagnostique et/ou thérapeutique. L'intervention percutanée, effectuée sous anesthésie locale ou générale est en effet préparée, guidée et contrôlée par l'image. Qu'il s'agisse de cardiologie, ou de radiologie interventionnelle, vasculaire ou non, l'essor des techniques interventionnelles guidées par rayons $\mathrm{X}$ dont l'intérêt thérapeutique ne se discute plus, conduit à des procédures de plus en plus longues et complexes délivrant des doses de rayons X qu'il est indispensable de maîtriser ${ }^{3}$. En effet, le risque d'apparition d'effets cutanés déterministes ne peut être écarté avec des procédures techniquement difficiles et longues. Il est fréquent de délivrer au cuir chevelu des doses de l'ordre de 5 Gy pour des embolisations d'anévrismes intra crâniens. En radiologie vasculaire périphérique, au niveau de la région abdominale, des procédures longues, avec des incidences obliques ou en profil strict chez des patients obèses peuvent être associées à des doses cutanées supérieures à 5 ou 6 Gy.

L'exposition prolongée des patients génère fréquemment une dose maximum à la peau supérieure à 2-3 Gy, fourchette de dose seuil admise d'apparition d'effets déterministes cutané radio-induits (d'un érythème précoce transitoire)

\footnotetext{
3 Rapport du groupe de travail sur la radioprotection en radiologie interventionnelle. Recommandations pour l'amélioration de la radioprotection en radiologie interventionnelle - GTRI/GPMED - juin 2010.
} 
(Roche, 2010). Au-delà de ce seuil, la gravité des lésions augmente avec la dose reçue, provoquant ainsi une épidermite sèche (10 Gy) ou une nécrose cutanée dans des cas extrêmes (18 Gy).

L'évaluation de la dose à la peau à partir des paramètres machine $(\mathrm{kV}, \mathrm{mAs}$, filtration) ou des indicateurs dosimétriques disponibles tels que le produit kerma surface (PKS) ou le kerma dans l'air (Ka) reste approximative. Plus précisément, le PKS permet de calculer une dose totale à la peau mais la corrélation avec la dose maximum à la peau est faible (Van de putte, 2000). Quand au Ka, il indique le kerma dans l'air pour un point de référence situé au centre du faisceau et à $60 \mathrm{~cm}$ du foyer du tube RX, et ne prend pas en compte le rayonnement rétrodiffusé par le patient. La dose sera donc sous ou surestimée suivant l'incidence du faisceau. La question de la recoupe des champs, fréquente en neuroradiologie avec l'utilisation d'incidences orthogonales, est également déterminante dans la problématique de la connaissance de la dose maximale à la peau, les PKS et Ka des installations ne prenant jamais en compte le chevauchement partiel des champs, à l'origine de « points chauds ». Ces constatations montrent que la mesure directe est préférable pour une évaluation fiable de la dose maximale à la peau. Dans cette optique, nous avons utilisé des films radiochromiques XR-RV3-Gafchromic ${ }^{\circledR}$ positionnés contre la peau des patients pendant des procédures radiologiques supposées à risque, afin de connaitre rapidement en fin d'examen la dose cutanée maximale et la cartographie de la distribution dosimétrique en fonction des différentes incidences utilisées. Ce type de film auto-développant a en effet montré ses avantages par rapport aux films radiographiques et la grande précision dosimétrique que l'on peut en extraire (Niroomand-Rad et al., 1998 ; Butson et al., 2003 ; Dini et al., 2005).

\section{Matériels et méthodes}

\subsection{Matériels}

\subsubsection{Films radiochromiques}

Les films utilisés lors de l'étude étaient des films Gafchromic ${ }^{\circledR}$ XR-RV3 (ISP) constitués de quatre couches principalement composées de carbone, d'hydrogène et d'oxygène. Les films sont alors considérés comme équivalents tissus. Leur utilisation est possible sur une gamme d'énergie allant de $30 \mathrm{keV}$ à $30 \mathrm{MeV}$ et pour une dose intégrée de $0,01 \mathrm{~Gy}$ à $30 \mathrm{~Gy}$. La stabilité du noircissement a été évaluée à 24 heures et un effet de la température a été reporté. L'étude proposée par McCabe (McCabe et al., 2011) nous indique que la réponse des films est 


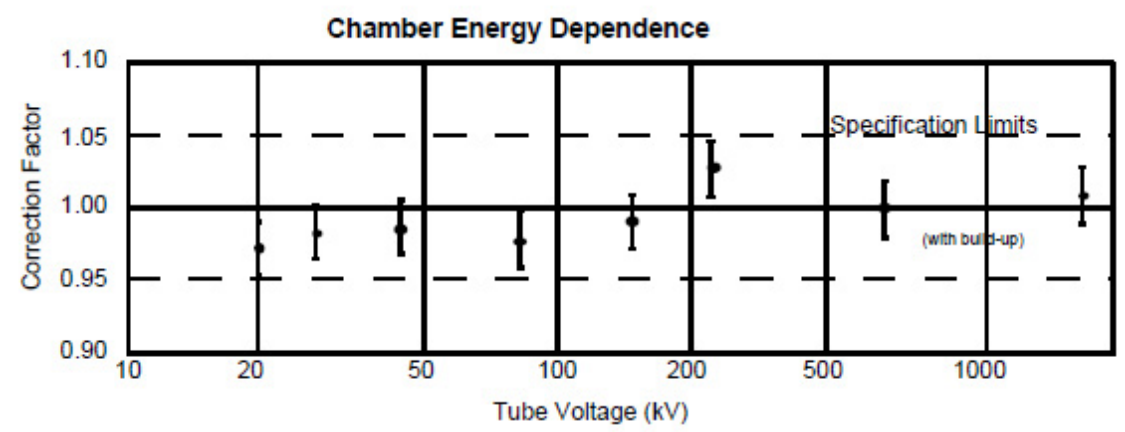

Figure 1 - Dépendance énergétique de la chambre d'ionisation. Energy dependence of the ionization chamber.

principalement dépendante de la qualité du faisceau et de son orientation (face orange ou blanche vers la source).

\subsubsection{Scanner à plat de lecture des films}

Le scanner à plat EPSON Expression 10000X avec une surface de $310 \times 437 \mathrm{~mm}^{2}$ (format A3) permet de numériser différents types de films par transmission ou par réflexion. Ce type de scanner est largement utilisé pour la dosimétrie par films (Ferreira et al., 2009). La résolution de sortie s'étend de 50 à 12800 dpi et les échelles de couleur de 24 ou 48-bit sont disponibles. La séparation des images en trois couleurs, correspondantes aux trois canaux du capteur CCD, rouge, vert, bleu (RGB), permet donc d'obtenir 255 ou 63535 niveaux de couleur (8 ou 16-bit par couleur). Les images obtenues ont été sauvegardées au format TIFF.

\subsubsection{La chambre d'ionisation Radcal ${ }^{\circledR} 10 \times 6-60 E$}

La chambre d'ionisation utilisée était une chambre 10x6-60E, étalonnée au Physikalish-Technishe Bundesansalt (PTB). L'incertitude globale de mesure de la dose est évaluée par le fabricant à $\pm 5 \%(k=2)$. La figure 1 montre que la réponse de la chambre 10x6-60E est peu dépendante de l'énergie sur une gamme de tensions du tube allant de 20 à plus de $1000 \mathrm{kV}$.

Dans le cadre de notre étude (tensions comprises entre 80 et $120 \mathrm{kV}$ ) nous avons considéré que la réponse de la chambre était indépendante de l'énergie et aucun facteur de correction n'a de ce fait été appliqué. 


\subsubsection{Le logiciel ImageJ et Lab Fit Curve Fitting Software}

ImageJ est un logiciel libre d'analyse et de traitement d'images.

Le logiciel Lab Fit permet d'obtenir une fonction analytique de la courbe sensitométrique à partir des points expérimentaux. La fonction obtenue a ensuite été rentrée dans ImageJ pour la conversion en dose de l'image.

\subsection{Méthodes}

\subsubsection{Scanner à plat}

Pour étudier la variation de la réponse du scanner en fonction du nombre de lectures, nous avons scanné cinq fois de façon successive un morceau de film exposé $(3 \mathrm{~cm} \times 3 \mathrm{~cm})$ à une dose de $1 \mathrm{~Gy}$. L'uniformité de lecture du scanner a été évaluée en scannant un film non exposé et en comparant les valeurs moyennes des pixels obtenues dans une région d'intérêt rectangulaire placée à différents endroits sur le film. La différence maximale observée est de 2,2 \%.

\subsection{2. Étude préliminaire}

L'évolution temporelle du noircissement des films a été étudiée en exposant quatre morceaux de films $(3 \mathrm{~cm} \times 3 \mathrm{~cm})$ respectivement à $0,1 \mathrm{~Gy}, 1 \mathrm{~Gy}, 4 \mathrm{~Gy}$ et $10 \mathrm{~Gy}$ et lus toutes les 24 heures pendant 72 heures. Après irradiation, les films ont été conservés dans une enveloppe pour les protéger de la lumière. L'évolution maximale relevée était de $1,1 \%$. Les films Gafchromic ${ }^{\circledR}$ XR-RV3 ont été principalement lus 24 heures après la procédure radiologique.

Avant de réaliser l'étalonnage en dose, nous avons vérifié l'uniformité du champ d'irradiation à l'aide d'un film positionné sur une couche de polystyrène pour placer le système dans «l'air ». Le film obtenu montrait une distribution de la dose inférieure à $3 \%$ entre le centre et le bord du champ. L'uniformité du champ a été prise en compte pour le calcul d'incertitudes.

\subsection{3. Étalonnage des films}

Les films XR-RV3 ont été manipulés et découpés en suivant les recommandations du groupe de travail 55 de l'AAPM ${ }^{4}$ (température entre $20-25^{\circ} \mathrm{C}$ ). 26 morceaux de $3 \mathrm{~cm} \times 3 \mathrm{~cm}$ ont été découpés à partir d'un même film $(36 \mathrm{~cm} \times 43 \mathrm{~cm})$ et utilisés pour réaliser l'étalonnage. La distance foyer-film était de $50 \mathrm{~cm}$. Les valeurs du Kerma dans l'air, mesuré avec la chambre d'ionisation, des films

\footnotetext{
4 American Association of Physicists in Medicine.
} 
exposés étaient : tous les $0,1 \mathrm{~Gy}$ de 0,1 à $1 \mathrm{~Gy}$ puis tous les $0,5 \mathrm{~Gy}$ de 1 à $6 \mathrm{~Gy}$ et enfin par incrément 1 Gy jusqu'à $11 \mathrm{~Gy}$. Les films ont été disposés selon les recommandations du constructeur, coté actif (orange) face à la source de rayons X. Un film non exposé a été conservé pour permettre la mesure du voile de fond.

L'image 48 bits couleur obtenue a été traitée avec le logiciel d'analyse d'images ImageJ. Une image 48 bits couleur est composée d'une superposition de trois composantes primaires codées sur 16 bits : rouge, vert et bleu (mode RGB) qui peuvent être séparée avec ImageJ en trois images de profondeur de pixel 16 bits. Chaque couleur a une sensibilité différente et a été analysée séparément. Ce dispositif a été répété pour deux tensions du tube extrêmes utilisées en radiologie interventionnelle : 81 et $117 \mathrm{kVp}$ pour évaluer la réponse des films en fonction de l'énergie.

La composante rouge était la plus sensible au niveau des faibles valeurs de dose (Fig. 2). Pour l'étude de cas clinique, nous avons choisi de lire les films avec la composante rouge (plus sensible) jusqu'à 6 Gy (saturation) et avec la composante verte pour des doses supérieures. La courbe bleue restait difficilement exploitable car elle apparaissait très peu sensible.

Il existait peu d'écart entre les courbes d'étalonnage $81 \mathrm{kVp}$ et $117 \mathrm{kVp}$. L'écart moyen relevé entre les courbes était de 1,44\%, avec une différence maximale de 3,6\%. Même si l'écart observé était faible, nous avons pris en compte la courbe d'étalonnage se rapprochant le plus des énergies employées lors des examens. La fonction analytique fournie par le logiciel LabFit qui correspondait le mieux aux courbes sensitométriques était dans ce cas:

$$
Y=\frac{A+X}{B+C X}+D X
$$

avec $Y$ la dose, $X$ la valeur d'un pixel et $A, B, C, D$ les coefficients de l'équation.

L'incertitude globale a été estimée en fonction de la dose (propagation des incertitudes liée à la courbe sensitométrique). Celle-ci augmentait régulièrement avec des valeurs allant de $10 \%$ pour une dose de $2 \mathrm{~Gy}$, à $20 \%$ pour une dose de 12 Gy ( $8 \%$ pour une dose de $1 \mathrm{~Gy}, 15 \%$ pour une dose de $3 \mathrm{~Gy}, 16 \%$ pour une dose de 4 Gy, $21 \%$ pour une dose de 5 Gy et $20 \%$ pour une dose de 6 Gy, avec la composante rouge et entre 15 et $20 \%$ entre 7 et 12 Gy avec la composante verte).

\subsubsection{Processus de la mesure in-vivo par film radiochromique}

Les films radiochromiques doivent être disposés sur le patient pour coller le plus possible à la peau du patient (mesure du rayonnement rétrodiffusé) et il est important de connaître la balistique classique de la procédure pour entièrement 

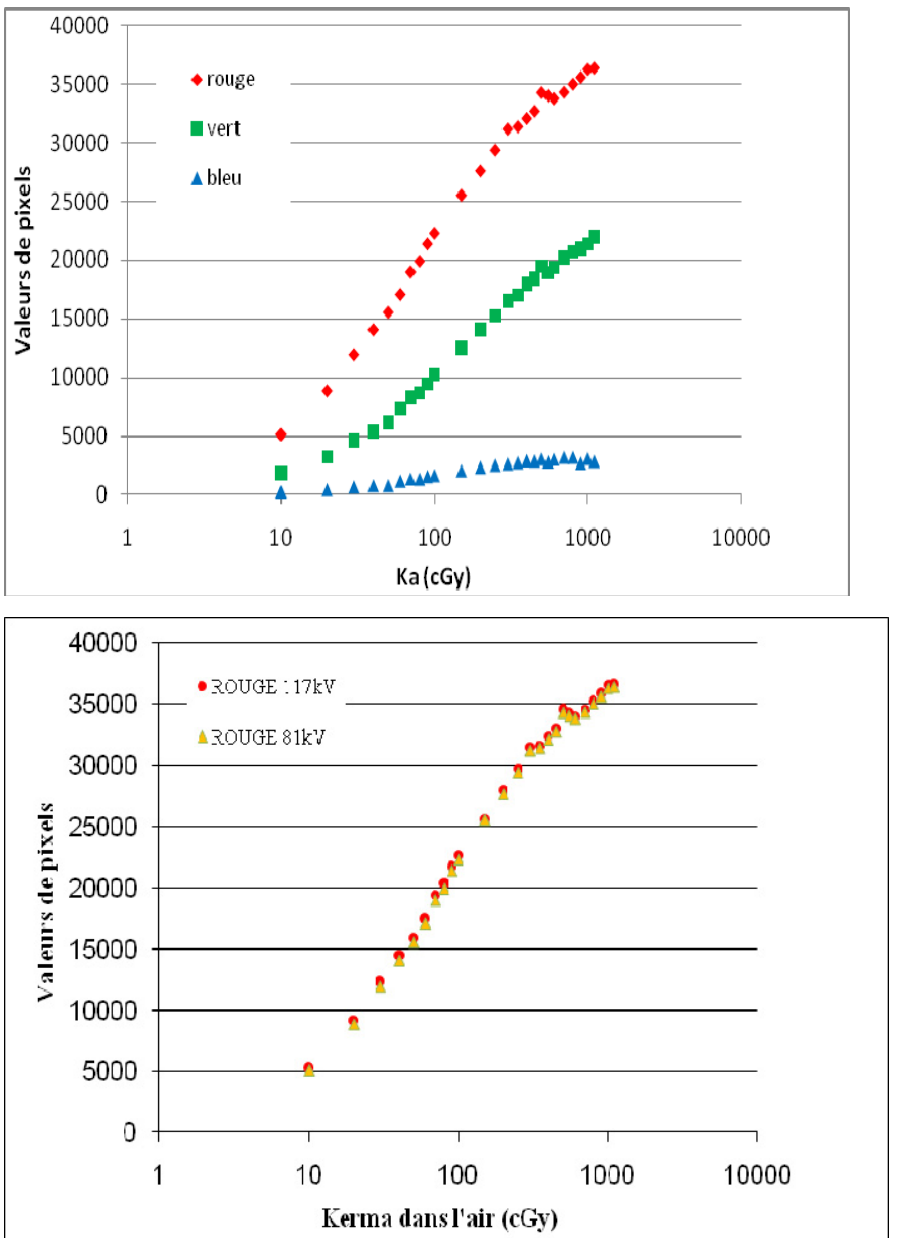

Figure 2 - Courbe sensitométrique pour les trois composantes de l'image (haut). Comparaison des courbes sensitométriques pour une tension de $81 \mathrm{kVp}$ et $117 \mathrm{kVp}$.

Sensitometric curve for the three components of the image (top). Comparison of sensitometric curves for a voltage of $81 \mathrm{kVp}$ and $117 \mathrm{kVp}$.

cartographier l'exposition. L'exploitation du film sur le logiciel ImageJ est réalisée en entrant la fonction représentative de la courbe sensitométrique. L'image finalement obtenue est donc une cartographie de la dose à la peau reçue par le patient. Cette image peut être exploitée avec différents outils pour effectuer des relevés dosimétriques (Fig. 3). 


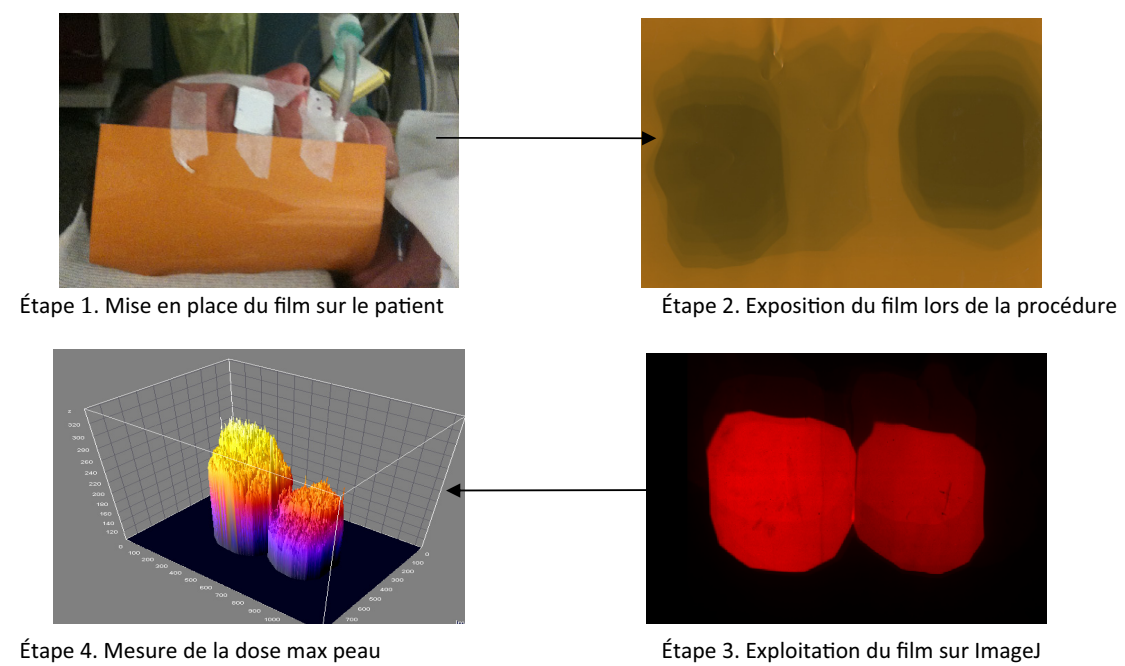

Figure 3 - Étapes nécessaires à l'obtention de la dose maximale à la peau. Steps required to obtain the maximum dose to the skin.

\subsection{5. Étude clinique : utilisation des films en pratique en neuroradiologie et radiologie vasculaire interventionnelle}

\subsubsection{En radiologie vasculaire interventionnelle}

Nous avons pu constater après enquête quotidienne de plusieurs mois que les interventions les plus irradiantes en radiologie vasculaire interventionnelle étaient les procédures abdomino-pelviennes. Nous avons donc étudié :

- 13 chimioembolisations hépatiques,

- 11 dilatations artérielles rénales,

- 7 embolisations hypogastriques.

\subsubsection{En neuroradiologie}

Dans ce domaine d'activité, les interventions les plus irradiantes étaient les embolisations d'anévrysmes intracrâniens et de malformations artério-veineuses (MAV). Nous avons donc étudié :

- 20 embolisations d'anévrysmes intracrâniens,

- $7 \mathrm{MAV}$. 
TABLEAU I

Récapitulatif des résultats obtenus : nombre de patients ayant reçu des doses supérieures à 3 Gy d'après les données machine et les films radiochromiques.

Sum up of results: the number of patients receiving doses greater than $3 \mathrm{~Gy}$ from the machine data and radiochromic films.

\begin{tabular}{llc}
\hline \multicolumn{1}{c}{ Dose-patient $>\mathbf{3}$ Gy } & Dose /Données machine (Ka / PKS) & Dose /Films radiochromiques \\
\hline $\begin{array}{l}\text { Radiologie vasculaire } \\
\text { interventionnelle } \\
\text { (31 cas étudiés) }\end{array}$ & 4 patients & 6 patients \\
$\begin{array}{l}\text { Neuroradiologie } \\
(27 \text { cas étudiés) }\end{array}$ & $\begin{array}{l}\text { 0 patients (Uniquement PKS disponible à la } \\
\text { console, insuffisant à lui seul pour évaluer } \\
\text { la dose reçue) }\end{array}$ & 6 patients \\
\hline $\begin{array}{l}\text { Total } \\
(58 \text { cas étudiés })\end{array}$ & 4 patients & 12 patients \\
\hline
\end{tabular}

\section{Résultats}

Les films radiochromiques ont permis d'identifier 12 patients, sur 58 cas étudiés (Tab. I), pour lesquels la dose dépassait le seuil de 3 Gy (seuil des effets déterministes). L'utilisation du Kerma dans l'air et du produit Kerma Surface fourni par les installations n'auraient permis de déceler que 4 d'entre eux.

\subsection{En radiologie vasculaire interventionnelle}

Nous prenons ici l'exemple d'une dilatation rénale. Le Kerma dans l'air $\left(K_{a}\right)$ fourni par l'installation ( $4,4 \mathrm{~Gy}$ ) était très différent de la dose maximale relevée sur le film radiochromique $(6,8 \mathrm{~Gy} \pm 1,4 \mathrm{~Gy})$. L'écart observé de $35 \%$ résulte du rayonnement rétrodiffusé par le patient pris en compte uniquement par le film.

\subsection{En neuroradiologie}

L'image dosimétrique d'une procédure d'embolisation d'anévrisme nous a permis de constater que la dose maximale reçue résultait de l'irradiation provoquée par l'incidence latérale $\left(\mathrm{PKS}=265131 \mathrm{mGy} . \mathrm{cm}^{2}\right)$. D'après le PKS de l'installation et les dimensions du champ $(12 \mathrm{~cm} \times 12 \mathrm{~cm})$, le Kerma dans l'air calculé était de $1,84 \mathrm{~Gy}$. Avec ImageJ nous avons obtenu une dose de 3,2 Gy $( \pm 0,47 \mathrm{~Gy})$ soit une différence de $42 \%$ entre les deux doses. Le PKS fourni par l'installation considère une répartition uniforme de la dose sur l'ensemble du champ et une superposition de tous les champs, ce qui expliquait la différence relevée entre les deux doses. 


\section{Discussion}

\section{L'intérêt de l'utilisation des films radiochromiques dans notre étude}

Durant cette étude, sur 31 procédures étudiées en radiologie vasculaire, les seuls contrôles des paramètres usuels observés (PKS et Ka) auraient permis de déceler 4 patients pour lesquels la dose maximale à la peau apparaissait supérieure à $3 \mathrm{~Gy}$. L'utilisation des films radiochromiques durant cette même période a permis d'identifier 2 patients supplémentaires pour lesquels une dose supérieure à 3 Gy a été mesurée, et justifiant l'orientation vers un suivi médical particulier. En neuroradiologie, le seul paramètre-machine disponible était le PKS, et il ne permettait pas à lui seul d'estimer la dose reçue à la peau du patient. En l'absence de films radiochromiques, il n'y avait pas d'évaluation de la dose reçue pour les patients de neuroradiologie interventionnelle. Sur 27 procédures étudiées, l'utilisation des films a permis de déceler 6 patients ayant reçu des doses à la peau supérieures à $3 \mathrm{~Gy}$. Par ailleurs les films radiochromiques, insensibles à la lumière visible sont d'utilisation facile en routine et peuvent lus dès la fin des examens.

\section{Les limites de l'utilisation des films radiochromiques}

Pour une bonne efficacité, les films doivent être positionnés avec le côté actif face à la source, mais aussi et surtout bien disposés sous le patient. Ils doivent couvrir l'ensemble de la zone à explorer de sorte à ne pas couper (ou manquer) les champs d'irradiation utilisés lors de l'intervention. Leur conservation dans une enveloppe noire et dans une pièce à température ambiante $\left[\sim 25^{\circ} \mathrm{C}\right]$ est indispensable pour leur bon fonctionnement.

Les films Gafchromic ${ }^{\circledR}$ XR-RV3 fournissent une mesure in-vivo différée de la procédure et l'opérateur n'est pas averti sur la dose maximale à la peau, en cours de procédure.

Enfin, sur le crâne en particulier, ils n'épousent jamais parfaitement les contours anatomiques du patient; il existe donc des zones où le film n'est pas plaqué contre la peau et pour lesquelles le rayonnement retrodiffusé pourrait être sous estimé.

\section{Incertitudes sur la dose mesurée avec les films radiochromiques}

Les patients pour lesquels nous avons mesuré une dose égale à $3 \mathrm{~Gy}( \pm 0,45 \mathrm{~Gy})$ ont donc reçu entre 2,55 et 3,45 Gy, soit une incertitude de l'ordre de $15 \%$. Plus élevée que dans le domaine de la radiothérapie, cette incertitude reste néanmoins compatible avec l'utilisation souhaitée. En effet, c'est une estimation de la dose 
reçue qui est avant tout recherchée, pour identifier les patients ayant dépassé le seuil de 3 Gy et nécessitant un suivi dermatologique.

\section{Dosimétrie patient en radiologie interventionnelle : perspectives}

Les examens de radiologie interventionnelle sont en règle générale des procédures dont l'exposition aux rayonnements $\mathrm{X}$ est contrôlée, mais quelques unes de ces interventions peuvent atteindre d'importants temps de fluoroscopie, de nombreuses séquences de radiographie, et donc des doses significatives. Le risque pour le patient de développer des effets radio-induits existe, mais doit bien sûr être apprécié en fonction du risque évolutif de la pathologie en cause. L'utilisation des films permet, grâce à la mesure de cette dose, d'anticiper la connaissance des effets radio-induits et de diriger le patient vers un suivi médical adapté, qui s'intègre dans un contexte global de radioprotection des patients, dont l'axe central est l'optimisation. Les interventions abdomino-pelviennes en radiologie vasculaire interventionnelle et les embolisations d'anévrysmes intracrâniens et de malformations artério-veineuses en neuroradiologie ont été identifiées comme des procédures pouvant délivrer dans certains cas des doses supérieures à $3 \mathrm{~Gy}$ (seuil à partir duquel l'ASN demande un suivi médical particulier). La littérature (Koenig et al., 2001) fait état de l'apparition d'effets cutanés plus ou moins graves consécutifs à des procédures radiologiques interventionnelles et la technologie des films radiochromiques apparaît comme une solution concrète et fiable pour l'évaluation de la dose à la peau.

\section{Conclusion}

Les effets cutanés consécutifs à l'exposition aux rayons $\mathrm{X}$ apparaissent pour des doses supérieures à 2-3 Gy. Les installations actuelles ne permettent pas de connaître précisément cette information dosimétrique. Une méthode de mesure simple de la dose absorbée cutanée délivrée au patient, afin de prévoir pour quels patients la surveillance des plans cutanées exposés doit être assurée, est donc nécessaire. L'étude présentée confirme le besoin d'une évaluation en routine des doses reçues à la peau des patients pour les procédures interventionnelles vasculaires abdomino-pelviennes et pour les embolisations d'anévrysmes intracrâniens et des malformations artério-veineuses intracrâniennes en neuroradiologie interventionnelle, qui pouvaient générer des doses cutanées dépassant le seuil de 2-3 Gy.

Sur 58 procédures étudiées et considérées à risque, les seuls contrôles des paramètres usuels observés (PKS et Ka) ont permis de déceler 4 patients pour lesquels la dose à la peau apparaissait supérieure à $3 \mathrm{~Gy}$. L'utilisation des films radiochromiques a permis d'identifier 8 patients supplémentaires pour lesquels 
une dose supérieure à $3 \mathrm{~Gy}$ a été mesurée, justifiant l'orientation vers un suivi médical particulier.

\section{RÉFÉRENCES}

Butson M.J. et al., (2003) Radiochromic film for medical radiation dosimetry, Mat. Sci. Engineer. 41, 61-120.

Dini S.A. et al. (2005) Dosimetric evaluation of GAFCHROMIC ${ }^{\circledR}$ XR type T and R films, J. Appl. Clin. Med. Phys. 6 (1), 114-134.

Ferreira B.C., Lopes M.C., Capela M. (2009) Evaluation of an Epson flatbed scanner to read Gafchromic EBT films for radiation dosimetry, Phys. Med. Biol. 54, 1073-1085.

Koenig T.R., Wolff D., Mettler F.A., Wagner L.K. (2001) Skin injuries from fluoroscopically guided procedures, Am. J. Roentgenology 177.

McCabe B.P. et al. (2011) Calibration of XR-RV3 film for skin dose measurement, Med. Phys. 38 (4), 1919-1930.

Niroomand-Rad A., Blackwell C.R., Coursey B.M., Gall K.P., Galvin J.M., McLaughlin W.L., Meigooni A.S., Nath R., Rodgers J.E., Soares C.G. (1998) Radiochromic film dosimetry: Recommendations of AAPM Radiation Therapy Committee Task Group 55, Med. Phys. 25, 2093-2115.

Roche A. (2010) Radioprotection du patient en radiologie interventionnelle, J. Radiol. 91, 1231-1235.

Van de putte S. et al. (2000) Correlation of patient skin doses in cardiac interventionnal radiology with dose-area product, Brit. J. Radiol. 73, 504-513. 